\section{International Scientific Journal Theoretical \& Applied Science}

\author{
p-ISSN: 2308-4944 (print) e-ISSN: 2409-0085 (online) \\ Year: $2016 \quad$ Issue: $6 \quad$ Volume: 38 \\ Published: $30.06 .2016 \quad$ http://T-Science.org
}

SECTION 4. Computer science, computer engineering and automation.
Murat Sauranbayevich Tulenbayev Doctor of Technical Sciences, professor of Computer science, Dean of the Faculty Taraz State University named after M.Kh. Dulaty, Kazakhstan mtulenbaev@mail.ru

Svetlana Temirhanovna Beglerova Candidate of Science, Head of Sector Management of the Educational Process in the Distance Learning Department (DLD)

Taraz State University named after M.Kh. Dulaty, Kazakhstan sbeglerova@mail.ru

Marat Duisenkulovich Manapbaev Master of Engineering science, Software Engineer DLD Taraz State University named after M.Kh. Dulaty, Kazakhstan $\underline{\text { mhclon@mail.ru }}$

Zhanat Sauranbayevich Tulenbayev Doctor of Technical Sciences, professor of Power industry,

Taraz State University named after M.Kh. Dulaty,

Kazakhstan tulenbaev@mail.ru

\title{
DIGITAL FILTERING AND WAVELET PROCESSING OF SIGNALS OF CHEMICAL ANALYTICAL SYSTEMS
}

\footnotetext{
Abstract: There are cosidered questions of spectral representation and processing of signals of chemicalanalytical complexes with using of wavelet-technologies

Key words: chemical-analytical complexes, wavelet-technologies, synthesis of orthogonal basis

Language: Russian

Citation: Tulenbayev MS, Beglerova ST, Manapbaev MD, Tulenbayev ZS (2016) DIGITAL FILTERING AND WAVELET PROCESSING OF SIGNALS OF CHEMICAL ANALYTICAL SYSTEMS. ISJ Theoretical \& Applied Science, 06 (38): 74-77.

Soi: http://s-o-i.org/1.1/TAS-06-38-15 Doi: crossef http://dx.doi.org/10.15863/TAS.2016.06.38.15

\section{ЦИФРОВАЯ ФИЛЬТРАЦИЯ И ВЕЙВЛЕТ ОБРАБОТКА СИГНАЛОВ ХИМИКО-АНАЛИТИЧЕСКИХ КОМПЛЕКСОВ}

Аннотация: Рассматриваются вопросы спектрального представления и обработки сигналов химикоаналитических комплексов, с использованием вейвлет технологий.

Ключевые слова: химико-аналитические комплексы, вейвлет технологии, синтез ортогонального базиса.
}

Современные аналитические приборы: хроматографы, спектрофотометры, рентгенофлуориметры и др. определяют состав наземных экоаналитических лабораторий экологического мониторинга. В настоящее время приборостроители предприняли переход от производства аналитических приборов к серийному выпуску многоцелевых компьютеризованных химикоаналитических комплексов (ХАК) типа «ИНЛАН»
(ИНЛАН - сокращенно от интеллектуального лабораторного анализа). ХАК - определяется как сертифицированная совокупность материальной (средства измерений, вычислительная техника, стандартные образцы, вспомогательное оборудование и т.п.), а также интеллектуальной (методики, программный продукт, эргономика) составляющих химического анализа [1]. Таким образом, в одном комплекте системно объединены 
непосредственно приборы, программноматематическое обеспечение (ПМО), средства метрологического обеспечения, комплект методик анализа и все периферийные устройства, необходимые для реализации этих методик. Аналитические комплексы не просто позволяют заменить одни приборы на другие, более совершенные, а создают в экоаналитических лабораториях новую технологическую среду, позволяющую превратить химико-аналитические лаборатории в подобие производственных участков, дающих быстро и дешево химикоаналитическую информацию. Существенного эффекта повышения качества аналитических измерений с наименьшими затратами ресурсов достигается за счет использования соответствующего ПМО. Таким образом, вопросы разработки новых алгоритмов ПМО, как наиболее динамичной составляющей ХАК, остаются актуальными.

Значительный неослабевающий интерес в мире к представлению сигналов в базисе всплесков (wavelets) обусловлен главным образом их приложениями к проблемам сжатия и обработки информации (аудио и графической). В настоящее же время построение новых базисов с нужными свойствами можно считать требующим определенных навыков ремеслом. Аппарат базисов всплесков оказался одинаково продуктивен, как для чисто теоретических вопросов, так и для прикладных. Оказалось, что существует множество базисов, хорошо локализованных по пространству, чьи преобразования Фурье также хорошо локализованы. При этом оказалось, что степенью локализации во временной и частотной области можно управлять, что дает возможность получить своеобразную интерполяцию между представлением функции (сигнала) во временной (или пространственной) области и ее представлением в частотной области, т.е. ее представлением через преобразование Фурье [2,3].

Обобщением гармонического анализа является применение для представления сигнала $S(t)$ в виде ряда или интеграла других систем функций. Такими функциями, в принципе могут быть любые системы линейно независимых функций $\{g(k, t), k=1,2, \ldots, N\}$ и тогда сигнал $S(t)$ можно представить в виде:

$$
\begin{gathered}
S(t)=\sum_{k=1}^{N} S(k) g(k, t) \\
S(k)=\int_{T} S(t) G(k, t) d t=\int_{T} S(t) \sum_{i=1}^{N} B_{k, i} g(i, t) d t
\end{gathered}
$$

Функции $g(k, t)$ называются базисными, а $G(k, t)$ - взаимными (дуальными) базисными функциями:

$$
G(k, t)=\sum_{i=1}^{N} B_{k, i} g(i, t)
$$

Дискретное преобразование выходного сигнала аналитического прибора $y(t)$ будем рассматривать как разложение вида (1) непрерывного сигнала на конечном интервале времени по системе непрерывных функций $\{g(k, t)\}$. Совокупность коэффициентов такого разложения является дискретным аналогом сигнала - его спектром.

1. Проектирование вейвлетов для обработки сигналов ХАК, специальные условия[4-7].

Существует значительное количество систем базисных функций, удовлетворяющих общим требованиям, предъявляемым к базисам и обеспечивающим необходимое разложение $y(t)$ [3]. Однако из всего их многообразия целесообразно выбрать такие функции, которые отвечали бы некоторым дополнительным условиям, вытекающим из характера обработки выходного сигнала аналитического прибора. Это, прежде всего [4]:

a) повышение отношения сигнал/помеха;

б) некоррелированность коэффициентов разложения;

в) улучшение разделения полезных компонентов сигнала.

Выполнение этих требований позволило бы упростить алгоритмы обработки и улучшить качество получаемых оценок.

Обозначим такую систему базисных и взаимных базисных функций через $\{\varphi(k, t), \Phi(k, t)\}$. Чтобы в пространстве, натянутом на базисные функции, компоненты сигнала были различимыми (желательно - разделенными), базисные функции должны зависеть от существенного параметра $l$. В дискретном представлении сигнала, система базисных функции в качестве аргумента содержит квантованные значения параметра $l=k \Delta l$. Величина $\mathrm{k}$ определяет номер функции и введена в ее обозначение в виде параметра.

Максимальное отношение сигнал/помеха в спектральной области предполагает, что каждая взаимная базисная функция $\Phi(k, t)$, с помощью которой по выражению, аналогичному (2), вычисляется спектр сигнала $S(k)$, должна удовлетворять уравнению согласованной фильтрации:

$$
\int_{T} B(t, \tau) \Phi(k, \tau) d \tau=f(k, t)
$$

где $f(k, t)$ - модель сигнала, у которого некоторый (существенный) параметр $\quad l=k \Delta l$ : $\Delta l$-шаг квантования по оси $l$.

Из (4) следует, что функции $\{f(k, t)$, $k=1,2, \ldots, N\}$, так же как и функции $\Phi=(k, t)$, должны образовывать упорядоченную по значениям $k \Delta l$ систему (т.е. образованы сдвигом $\Delta l$ модели сигнала по оси $l$ ), при фиксированном на некотором уровне (точнее - на уровне параметров сигнала) векторе остальных параметров. Как 


\begin{tabular}{|c|c|c|c|c|c|c|}
\hline Impact Factor: & $\begin{array}{l}\text { ISRA (India) } \\
\text { ISI (Dubai, UAB } \\
\text { GIF (Australia) } \\
\text { JIF }\end{array}$ & $\begin{array}{r}=1.344 \\
=0.829 \\
=0.564 \\
=1.500\end{array}$ & $\begin{array}{l}\text { SIS (USA) } \\
\text { PИHЦ (Russia) } \\
\text { ESJI (KZ) } \\
\text { SJIF (Morocco) }\end{array}$ & $\begin{array}{l}=0.912 \\
=0.234 \\
=1.042 \\
=2.031\end{array}$ & $\begin{array}{l}\text { ICV (Poland) } \\
\text { PIF (India) } \\
\text { IBI (India) }\end{array}$ & $\begin{array}{l}=6.630 \\
=1.940 \\
=4.260\end{array}$ \\
\hline
\end{tabular}

правило, вследствие конечной длительности сигнала существенный параметр входит в модель, описывающую сигнал, нелинейно. Поэтому система функции $\{f(k, t)\}$ будет линейно независимой.

Некоррелированность коэффициентов достигается при каноническом разложении случайного процесса. Каноническое разложение процесса $y(t)$ с корреляционной функцией $B(t, \tau)$ обеспечивается, если базисные функции $\{\varphi(k, t), \Phi(k, t)\}$ удовлетворяют уравнению:

$$
\varphi(k, t)=\frac{1}{\sigma_{k}^{2}} \int_{T} B(t, \tau) \Phi(k, \tau) d \tau
$$

где

$$
\sigma_{k}^{2}=\iint_{T} B(t, \tau) \Phi(k, t) \Phi(k, \tau) d t d \tau
$$

$\sigma_{k}^{2}$ имеет смысл дисперсии $k$-ой спектральной составляющей.

Сравнивая выражения (5) (6), можно видеть, что для удовлетворения обоих вышеуказанных требований между функциями $f(k, t)$ и базисом $\varphi(k, t)$ должна существовать линейная зависимость:

$$
f(k, t)=\sigma_{k}^{2} \varphi(k, t)
$$

Тогда по (5), взаимный базис $\Phi(k, t)$ будет равен:

$$
\Phi(k, t)=\frac{1}{\sigma_{k}^{2}} \sum_{i=1}^{N} v_{k i} f(i, t)
$$

Подставляя в это выражение $f(i, t)$ из (4), получим

$$
\Phi(k, t)=\frac{1}{\sigma_{k}^{2}} \sum_{i=1}^{N} v_{k i} \int_{T} B(t, \tau) \Phi(i, \tau) d \tau
$$

Следовательно, базисная система функций $\{\varphi(k, t) ; \Phi(k, t)\}, \quad$ позволяющая обеспечить согласованную фильтрацию и некоррелированность спектральных отсчетов, должна удовлетворять уравнениям (8) и (9). В частности, искомым решением является базисная система, образованная собственными функциями уравнения

$$
\int_{T} B(t, \tau) \Phi(i, t) d \tau=\lambda_{i} \Phi(i, t)
$$

Такая базисная система предполагает разложение, известное как разложение КаруненаЛоэва [2]. При этом $\vartheta_{k, i}$ образуют диагональную матрицу с элементами $\vartheta_{k k}=\lambda_{k}^{-1} \sigma_{k}^{2}$. Это приводит, как следует из (8) и (9), к тому, что взаимный базис оказывается линейно связанным с моделью сигнала $f(k, t)$, а значит, система функций

$\{f(k, t)\}$ должна образовывать взаимный базис.

Однако реальные сигналы аналитических приборов, а значит и их модели, таким свойством не обладают.

Таким образом, построить базисную систему, удовлетворяющую одновременно всем требованиям, невозможно и имеет смысл рассматривать возможность построения базисных систем, близких к оптимальным.

Ортогональные системы базисных функций, предназначенные для обработки выходных сигналов ХАК и, в совокупности, удовлетворяющие перечисленным требованиям (а-в), назовем вэйвлет базисными системами (ВБС).

2. Синтез вейвлетных систем, удовлетворяющих условию некоррелированности и максимизации отношения сигнал/помеха в спектральных отсчетах [8-10].

Рассмотрим синтез ортогонального базиса, близкого к оптимальному базису Карунена-Лоэва, отвечающего требованиям некоррелированности и максимизации отношения сигнал/помеха спектральных составляющих сигнала. Для этого введем систему линейно-независимых функций $\{U(k, t)\}$ удовлетворяющих уравнению, аналогичному (4):

$$
\int_{T} B(t, \tau) U(k, \tau) d \tau=f(k, t)
$$

и на их основе построим систему базисных функций $\{\varphi(k, t) \Phi(k, t)\}$ удовлетворяющих условию (5), воспользовавшись процедурой, аналогичной ортогонализации по Грамму-Шмидту [3]. Положим, что $\Phi(1, t)=U(1, t)$. Тогда $\varphi(1, t)$ вычисляется по (5) и (6) при $k=1$ и известной $\Phi(1, t)$ :

$$
\varphi(1, t)=\frac{1}{\sigma_{1}^{2}} \int_{T} B(t, \tau) \Phi(1, \tau) d \tau
$$

затем полагаем, что

$$
\Phi(2, t)=U(2, t)-\gamma_{21} \Phi(1, t),
$$

где из обеспечения условия взаимной ортогональности $\Phi(2, t)$ и $\varphi(1, t)$ коэффициент $\gamma_{21}$ выбирается равным:

$$
\gamma_{21}=\int_{t} U(2, t) \varphi(1, t) d t=\frac{1}{\sigma_{1}^{2}} \int_{t} f(2, t) \Phi(1, t) d t
$$

Вторая функция $\varphi(2, t)$ находится аналогично по (5) и (6) при $k=2$ и известной $\Phi(2, t)$. Продолжая 
процедуру далее, получим для $k$-ой функции $\Phi(k, t)$ выражение:

$$
\Phi(k, t)=U(k, t)-\sum_{k=\tau}^{k-1} \gamma_{k \tau} \Phi(\tau, t)
$$

где

$$
\gamma_{k \tau}=\int_{T} U(k, t) \varphi(\tau, t)=\frac{1}{\sigma_{2}^{2}} \int_{T} f(k, \tau) \Phi(\tau, \tau) d \tau, \mathrm{k}>\tau
$$

a $\varphi(\tau, t)$ определяется по (5) с учетом (6), (11).

Отметим что в случае комплексной системы функций $\{U(k, t)\}$ получаем комплексный базис и в (12) следует подставлять вместо $\varphi(\tau, t)$ комплексно-сопряженную с ней функцию $\varphi^{*}(\tau, t)$. Если система функций $\{U(k, t)\}$ ортогональна, то, естественно, $\gamma k \tau=0$ и $\Phi(k, t)=U(k, t)$. Иногда удобно иметь не рекуррентное, как (11), а явное выражение функций $\Phi(k, t)$ через функции $U(\tau, t)$. При этом $\Phi(k, t)$ можно представить в виде:

$$
\Phi(k, t)=U(k, t)-\sum_{\tau=1}^{k} d_{k \tau} U(\tau, t)
$$

где

$$
\begin{gathered}
d_{k \tau}=-\left(\gamma_{k \tau}-\sum_{i=\tau}^{k-1} \gamma_{k i} d_{i \tau}\right), \tau=\overline{1, k-2} \\
d_{k, k-1}=-\gamma_{k, k-1} ; d_{k k}=1
\end{gathered}
$$

Из приведенной процедуры вытекает, что выбор функций $\{\mathrm{U}(\mathrm{k}, \mathrm{t}), k=\overline{1, N}\}$ однозначно определяет искомую базисную систему. Учитывая специфику ее определения, назовем эту систему вэйвлет базисной системой (ВБС) и в дальнейшем, если нет специальных оговорок, под ВБС будем понимать базисные системы, построенные по изложенной методике.

Таким образом, $\varphi(k, t)$ и $\Phi(k, t)$ на основании (5), (11) и (13) будут иметь вид:

$$
\begin{gathered}
\Phi(k, t)=U(k, t)-\sum_{k=\tau}^{k-1} \gamma_{k \tau} \Phi(\tau, t)=\sum_{k=\tau}^{k-1} d_{k \tau} U(\tau, t) \\
\varphi(k, t)=\frac{1}{\sigma_{k}^{2}} \int_{T} B(t, \tau) \Phi(k, \tau) d \tau=\frac{1}{\sigma_{k}^{2}} \sum_{\tau=1}^{k} d_{k \tau} f(\tau, t)
\end{gathered}
$$

где $\varphi_{k \tau}$ и $d_{k \tau}$ определяется по (12) и (14) а $U(k, t)$ является решением уравнения (10).

В заключение отметим, что именно учет специфики коэффициентов $d_{k \tau}$ позволяет оценить преимущества вейвлет представления и предложить быстрые и экономные алгоритмы обработки в спектральной области. Теоретически МНК оценки параметров сигналов в таких вейвлет представлениях должны приближаться к оптимальным, если предположения о моделях сигналов и шума соответствуют реалиям.

\section{References:}

1. Buzanovskii VA, Rizhnev VU, et al. (2000) Rossiiskie ekoanaliticheskie kompleksi // Ekologiya i promishlennost Rossii. №1, pp.4-9.

2. Petuhov AP (1999) Vvedenie v teoriiu bazisov vspleskov. - SPb:Izd-vo SPbGTU, - 132p.

3. Novikov LV (2007) Obrabotka signalov na osnove ortonormirovannih kvaziveivletov // Priborostroenie: Izv. Vuzov. T.50, №1. pp.3-10.

4. Tulenbaev MS (2008) Modelirovanie signalov himiko-analiticheskih kompleksov ekologicheskogo monitoringa. //Vestnik TarGU imeni M.H. Dulati "Prirodopolzovanie i problemi antroposferi", - №3.- pp.291-294.

5. Tulenbaev MS (2009) Veivlet predstavlenie signalov himiko-analiticheskih kompleksov ekologicheskogo monitoringa // Nauka i inzhenernoe obrazovanie bez granic: trudi. mezhdunar. foruma (KazNTU, 13-14 november 2009) - Almati, 2009. - T. 2. - pp. 211-214.

6. Tulenbaev MS (2009) Veivlet-podobnie bazisi ortogonalnogo razlozheniya signalov $\mathrm{v}$ informacionnih sistemah ekologicheskogo monitoringa // VI Dulatovskie chteniya: mater.Mezhdunar. nauch.-prakt. konf. - Taraz, 2009 - CH. III. - pp. 51-54.
7. Tulenbaev MS, Rusinov LA (2010) Veivlet predstavleniya signalov analiticheskih kompleksov ekologicheskogo monitoringa // Dni nauki-2010: materiali VI Mezhdunar. nauch.-prakt. konf. CHehiya (march, 2010). Praga, 2010. - pp. 50-52.

8. Tulenbaev MS (2010) Spektralnie agoritmi obrabotki signalov himiko-analiticheskih kompleksov $\mathrm{v}$ geoinformacionnih sistemah ekomonitoringa. // Vestnik NAN RK - Almati, 2010 - №1 - pp.11-17.

9. Tulenbaev MS, Beglerova ST, Taszhurekova ZK (2011) Issledovanie algoritmov obrabotki ekoanaliticheskih dannih na osnove kompiuternogo imitaciionnogo modelirovaniya.// "Vestnik KazNTU im. K.I.Satpaeva" - Almati, №3 (85) - pp.227-231.

10. Tulenbaev MS, Beglerova ST, Taszhurekova ZK (2011) Vibor porozhdaiushih funkcii kvaziveivletov $\quad \mathrm{v}$ himiko-analiticheskih informacionnih sistemah ekologicheskogo monitoringa. // "Vestnik KazNTU im. K.I.Satpaeva", - Almati, - №3 (85) - pp.232238. 